\title{
Satepsi e a Qualidade Técnica dos Testes Psicológicos no Brasil
}

\author{
Lucila Moraes Cardoso ${ }^{1}$ \\ ${ }^{1}$ Universidade Estadual do Ceará, CE, Brasil
}

\author{
José Humberto da Silva-Filho ${ }^{2}$ \\ ${ }^{2}$ Universidade Federal do Amazonas, AM, Brasil
}

\begin{abstract}
Resumo: O presente artigo foi produzido mediante a comemoração dos 15 anos do Sistema de Avaliação dos Testes Psicológicos (Satepsi). Inicialmente serão apresentados aspectos históricos que fundamentam a decisão do Conselho Federal de Psicologia (CFP) de criar um sistema de análise dos instrumentos, bem como alguns dos desafios vivenciados pela comunidade científica nesses anos. Em seguida, a partir de uma revisão de estudos sobre os testes psicológicos disponíveis no Satepsi, pretende-se discutir a evolução das características dos testes psicológicos disponíveis no Brasil. Buscou-se contrastar os dados das pesquisas recentes com um levantamento feito em fevereiro de 2018 no Satepsi. No levantamento, foram identificados 180 testes psicológicos com pareceres favoráveis, sugerindo que do ponto de vista quantitativo praticamente quadruplicou o número de testes disponíveis no mercado desde a criação do Satepsi. Além disso, nota-se que a qualidade do material acompanhou os avanços da área. A partir das análises feitas, defende-se a importância do Satepsi para assegurar que materiais de qualidade estejam acessíveis para uso dos psicólogos.
\end{abstract}

Palavras-chave: Testes psicológicos, Psicometria, Satepsi.

\section{Satepsi and the Technical Quality of Psychological Testing in Brazil}

\begin{abstract}
This paper was produced to celebrate the $15^{\text {th }}$ anniversary of the Psychological Test Assessment System (in Brazil Satepsi). Initially, the historical aspects that underpin the Federal Council of Psychology's decision to create a system of analysis instruments will be presented, as well as some challenges faced by the scientific community in the past years. Thereafter, stemming from a review of carried out studies on the available psychological test in the Satepsi, the evolution of the available psychological tests' features in Brazil are discussed. Current research's data were contrasted with a survey performed in February, 2018, at Satepsi. 180 psychological tests with favorable positions were identified, suggesting that, from a quantitative point view, the number of available tests on the market virtually quadruplicated since the Satepsi creation. Furthermore, it was noticed that the quality of the material accomplished the advances in the area. Stemming from the carried out analysis, the importance of the Satepsi to assure that good materials will be reachable on use by psychologists is upheld.
\end{abstract}

Keywords: Psychological Testing, Psychometrics, Satepsi. 


\title{
Satepsi y la Calidad Técnica de las Pruebas Psicológicas en Brasil
}

\begin{abstract}
Resumen: El presente artículo fue producido mediante la conmemoración de los 15 años del Sistema de Evaluación de las Pruebas Psicológicas (en Brasil SATEPSI). Inicialmente van a ser presentados aspectos históricos que fundamentan la decisión del Consejo Federal de Psicología (CFP) de crear un sistema de análisis de los instrumentos, así como algunos de los desafíos vivenciados por la comunidad científica en estos años. Después, a partir de una revisión de los estudios sobre las pruebas psicológicas disponibles, se pretende discutir la evolución de las características de las pruebas psicológicas disponibles en Brasil. Se busca contrastar los dados de las investigaciones recientes con uno levantamiento hecho, en febrero de 2018, en SATEPSI. Fueron identificados 180 pruebas psicológicas con dictamen favorables, sugiriendo que del punto de vista cuantitativo prácticamente cuadruplicó el número de pruebas disponibles en el mercado desde la creación del SATEPSI. Además, se nota que la cantidad del material acompañó los avances del área. A partir de las análisis hechas, se defiende la importancia del SATEPSI para garantizar que los materiales de calidad estén accesibles para el uso de los psicólogos.
\end{abstract}

Palabras claves: Testes Psicológicos, Psicometría, Satepsi.

Os avanços na área da Avaliação Psicológica são crescentes (Primi, 2010; Noronha, \& Reppold, 2010; Santos, Anache, \& Santana, 2015). Entre os motivos do crescimento, pode-se elencar a criação do Sistema de Avaliação dos Testes Psicológicos (Satepsi), em 2001, pelo Conselho Federal de Psicologia (CFP), para certificar a qualidade dos instrumentos de avaliação psicológica que podem ser usados profissionalmente (Primi, \& Nunes, 2010).

O surgimento do Satepsi não ocorreu de forma isolada ou inesperada na história da Psicologia brasileira. No período de sua criação, a área da Avaliação Psicológica estava se recuperando de uma crise internacional, apontada por Anastasi ao assumir a presidência da American Psychological Association em 1967. Ela indicava que os psicólogos que lidavam com técnicas psicométricas estariam se afastando das correntes centrais da Psicologia contemporânea e que pareciam descartar seu conhecimento psicológico. Esse acontecimento levou outros autores a reiterar as críticas feitas por ela de que essa crescente dissociação estaria levando a área de Avaliação Psicológica à marginalização. A superação desta crise se deu a partir da década de 1980 nos Estados Unidos da América (EUA) e a partir da década de 1990 no Brasil (Hutz, \& Bandeira, 2003).

Concomitante à superação dessa crise, surgiram no Brasil, na década de 1990, as principais entidades da área da Avaliação Psicológica atuantes até o presente. São associações de professores, pesquisadores e profissionais da área de Avaliação Psicológica, tais como: a Associação Brasileira de Orientação Profissional (ABOP), em 1993; a Associação Brasileira de Rorschach e Métodos Projetivos (ASBRo), em 1993; e o Instituto Brasileiro de Avaliação Psicológica (IBAP), em 1997. Estas entidades foram fundadas em congressos científicos nacionais de suas áreas e se mantêm ativas até o presente, com a realização de congressos bianuais. Além disso, com objetivo de agregar as diversas demandas das entidades de Psicologia, foi fundada em 1997 o Fórum de Entidades Nacionais da Psicologia Brasileira (FENPB), que atualmente congrega 24 grandes entidades nacionais (FENPB, 2018).

A partir de uma visita aos sites das entidades, foram encontradas as seguintes informações: $\mathrm{A} A B O P$ realizou desde sua fundação até o presente oito congressos nacionais, a ASBRo está no seu nono congresso nacional, o IBAP realizou oito congressos brasileiros e o FENPB, que é responsável pela organização do Congresso Brasileiro de Psicologia (CBP), está na quinta edição. Além destes grandes eventos nacionais, destaca-se também o evento regional mineiro iniciado em 1993 (Encontro Mineiro de Avaliação Psicológica - EMAP) que, devido seu sucesso e prosperidade, já atinge uma dimensão quase nacional e está atualmente em sua 13a Edição. Esses congressos reúnem periodicamente professores, pesquisadores, profissionais e alunos que canalizam suas produções na área de Avaliação Psicológica para exposição. Para se ter 
uma dimensão da amplitude da produção científica atual, nos últimos congressos do IBAP e da ABOP, em 2017, foram apresentados, respectivamente, 569 e 255 trabalhos científicos e no último congresso da ASBRo, em 2016, foram apresentados 126 trabalhos. Todos estes trabalhos passaram por avaliações prévias por pares e são reconhecidos como tendo qualidade técnica para serem expostos nos eventos.

O impacto desse avanço da área também refletiu na ampliação dos Grupos de Trabalho (GTs) da Associação Nacional de Pesquisa e Pós-Graduação em Psicologia (ANPEPP), que facilita o encontro de pesquisadores e, consequentemente, da articulação das pesquisas das mais diversas áreas da Psicologia (Primi, 2010; Noronha, \& Reppold, 2010). No Simpósio de 2018, havia cinco GTs, a saber, GT 05 - Avaliação Cognitiva e Neuropsicológica (14 participantes), GT 06 - Avaliação em Psicologia Positiva e Criatividade (16 participantes), GT 07 - Avaliação Psicológica: Personalidade e Desenvolvimento Humano (12 participantes), GT 29 - Métodos Projetivos nos Contextos da Avaliação Psicológica (16 participantes) e GT 35 Pesquisa em Avaliação Psicológica (23 participantes) (ANPEPP, 2018).

Ao mesmo tempo em que a área da Avaliação Psicológica avançava com vigor, os próprios Conselhos Regionais de Psicologia (CRPs) e o CFP também recebiam inúmeros processos éticos disciplinares oriundos das práticas inadequadas da Avaliação Psicológica pelo Brasil afora. Além desses processos internos, o CFP também passou a receber representações judiciais de pessoas e categorias que alegavam terem sido prejudicadas por testes psicológicos, sobretudo em concursos, e requeriam que eles fossem extintos no Brasil.

Até o início dos anos 2000, realmente inexistia no Brasil qualquer controle de qualidade dos testes psicológicos e os pesquisadores da área já reconheciam a baixa qualidade técnica de muitos instrumentos disponíveis no mercado na época. No entanto, nos EUA já havia um sistema de revisão de testes, conceituado internacionalmente e até hoje considerado como referência, o Buros Institute of Mental Measurements, cuja primeira edição foi publicada por Oscar Buros em 1938. Embora se tivessem notícias que outros países também estivessem desenvolvendo seus próprios sistemas nacionais, a instituição do Satepsi colocou o Brasil como um dos pioneiros na implementação de um sistema de certificação baseado em critérios inter- nacionais de qualidade de todos os testes usados profissionalmente em um país (Primi, 2010).

De forma geral, pode-se dizer que os pesquisadores que criaram as entidades científicas de Avaliação Psicológica e os pesquisadores que a elas se associaram foram, praticamente, os mesmos atores que contribuíram para o fortalecimento da área de Avaliação Psicológica dentro do próprio CFP. Foram também os atores que - a muitas mãos - auxiliaram o CFP a editar a Resolução no 002/2003 e a criar o Satepsi. Esses mesmos atores trouxeram os fundamentos técnico-científicos de experiências internacionais consolidadas para a composição das normativas nacionais, como por exemplo: ITC Guidelines on Adapting Tests, da International Test Commission, 2000; Standards for Educational and Psychological Testing, da American Educational Research Association, American Psychological Association \& National Council on Measurement in Education, 1999; e, Guidelines for Educational and Psychological Testing, da Canadian Psychological Association, 1996 (Resolução CFP nº 002/2003).

Os avanços não tinham sido suficientes para diminuir as demandas judiciais de pessoas que alegavam serem prejudicadas por testes psicológicos utilizados por psicólogos e, com isso, percebia-se que a área ainda não estava pacificada. Um momento crítico experimentado pela categoria foi quando o Ministério Público Federal (MPF) moveu uma Ação Civil Pública em desfavor do CFP, em especial contra a recém-editada Resolução CFP no 002/2003. O MPF buscava tutela jurisdicional para exigir a abstenção de atos do CFP, por considerar que estes extrapolavam sua competência legal. O MPF pedia, liminarmente, que fosse proibida a comercialização e também a suspensão do uso de todo e qualquer teste psicológico no território nacional, com a apreensão dos produtos disponíveis no mercado, até que a União implementasse no Brasil os procedimentos para regularização dos testes psicológicos, pois considerava o CFP incompetente para tais ações. A sentença de Ação Improcedente, julgada pela Justiça Federal, confirmou o CFP como instância máxima de avaliação dos testes psicológicos no país sendo, portanto, o seu papel regulamentar esta prática no Brasil. Foi uma grande vitória da categoria, cuja luta foi coordenada pelo próprio CFP com o apoio de todas as entidades científicas da área (CFP, 2007).

A instituição do Satepsi consolidou um importante marco para área da Avaliação Psicológica no Brasil. Estavam instituídos os parâmetros normativos, compatíveis 
com os internacionais, para a categoria tanto para a produção científica como para o exercício da profissão na área de Avaliação Psicológica. Os congressos de todas as entidades nacionais, a partir de 2003 , rapidamente incorporaram os parâmetros da nova resolução do CFP. Em parte, porque estavam assimilados em sua prática e em parte porque passou a ser tema central no ensino da Avaliação Psicológica em todo Brasil.

Em 2012, o Instituto Brasileiro de Avaliação Psicológica resgatou a relevância da formação dos psicólogos e publicou as "Diretrizes para Ensino da Avaliação Psicológica". O documento estruturou-se em quatro tópicos, a saber, 1) "Competências em Avaliação Psicológica" que os profissionais devem desenvolver; 2) as "Disciplinas e Conteúdos Programáticos" fundamentais para uma boa formação; 3) a "Estrutura de Ensino" indicando a necessidade de criação de Laboratórios, testotecas, bibliografia atualizada e formação dos professores da área; 4) "Referências" bibliográficas atualizadas sugeridas para as disciplinas da área (Nunes et al., 2012).

Em 2015, o IBAP publicou o primeiro Catálogo dos Laboratórios de Avaliação Psicológica no Brasil, distribuídos por região do país, com informações sobre a data de fundação, pesquisadores, linhas de pesquisa e filiação institucional. Nesta publicação, foram identificados 46 Laboratórios e Centros de Estudos em Avaliação Psicológica no Brasil, indicando que a maioria deles foram criados após o Satepsi (IBAP, 2015).

A criação do Satepsi foi inicialmente questionada e até mesmo repudiada por muitos psicólogos, pois estes profissionais sentiam-se habilitados a manejar os instrumentos disponíveis que conheciam e mediante a retirada de circulação de vários deles pelo CFP, alegavam estarem sendo prejudicados no exercício da profissão. No entanto, a realidade dos instrumentos disponíveis à época indicava problemas graves, como citado por Noronha, Primi e Alchieri (2004). Em estudo realizado por estes autores com 146 testes publicados no Brasil por onze editoras, percebeu-se que $23,3 \%$ não traziam sequer a data de publicação e $76,7 \%$ foram publicados no Brasil entre 1920 a 1989, portanto muito antigos, e apenas $23 \%$ foram publicados entre 1990 a 2002. Dentre esses testes, muitos não apresentavam indicadores psicométricos adequados, pois foram identificados $48,6 \%$ com estudos de precisão, 52,7\% com estudos de validade e $43,8 \%$ com estudos de padronização. Com estudos de precisão, validade e padronização ao mesmo tempo foram identificados apenas $28,8 \%$.
Com o passar dos anos, os avanços que o sistema trouxe para a área foram reconhecidos. Para Primi e Nunes (2010), dentre os avanços, podem-se citar o estímulo para o desenvolvimento de pesquisas, reconhecimento da cientificidade que respalda a Avaliação Psicológica por outros setores nos quais os testes eram aplicados e um aumento expressivo na qualidade dos manuais de testes, que passaram a ser mais completos e detalhados do que até então.

Noronha e Reppold (2010) constataram que há diversas revisões sobre o estado da arte que evidenciam os avanços da área e da aplicação da Avaliação Psicológica em diferentes contextos. Ao fazer uma análise das produções da área, Primi (2010) apontou que no período entre 1985 até 1993 havia uma média de 32 publicações por ano. De fato, pesquisas publicadas antes de 2003 revelavam a precariedade dos manuais e dos instrumentos que eram utilizados naquele momento, seja de inteligência (Noronha, Sartori, Freitas, \& Ottati, 2001) ou de personalidade (Noronha, 2002).

No que tange ao reconhecimento científico dos instrumentos, há que se considerar o aumento na quantidade e diversidade de testes psicológicos disponíveis no mercado. Segundo Primi e Nunes (2010), no primeiro relatório do CFP, em 2004, havia 106 testes avaliados, sendo 51 desfavoráveis $(48,1 \%)$. Em 2010, foi reduzido em $12 \%$ o número de testes desfavoráveis. Dos 210 testes na lista, 76 estavam desfavoráveis ( $36,2 \%), 113$ favoráveis $(53,8 \%)$ e 21 em processo de análise (10\%). Em dezembro de 2015, o número de testes favoráveis aumentou para 158 (Reppold, Serafini, Ramires, \& Gurgel, 2017a) e 18 meses depois, em julho de 2017, totalizava 173 instrumentos favoráveis (Reppold, Serafini, Gurgel, \& Kaiser, 2017b; Reppold et al., 2017c). Em fevereiro de 2018, esse número atingiu 180 instrumentos com parecer favorável.

A Figura evidencia que dos 180 instrumentos disponíveis aos profissionais em fevereiro de 2018, 41 deles, ou $22,78 \%$, receberam parecer favorável em 2003, na época em que foi implantado o Satepsi. Nos últimos 15 anos quase quadruplicou a quantidade de testes aprovados disponíveis aos profissionais, evidenciando uma produção constante por parte dos pesquisadores brasileiros. A Resolução CFP no 009/2018 define que as normas técnicas dos testes devem ser revisadas a cada 15 anos. Portanto, em 2018, que muitos instrumentos precisam apresentar normas técnicas atualizadas, sendo possível que alguns deles - hoje favoráveis - passem por um período como desfavorável, até que seus autores e/ ou editoras os atualizem com novas normas. 


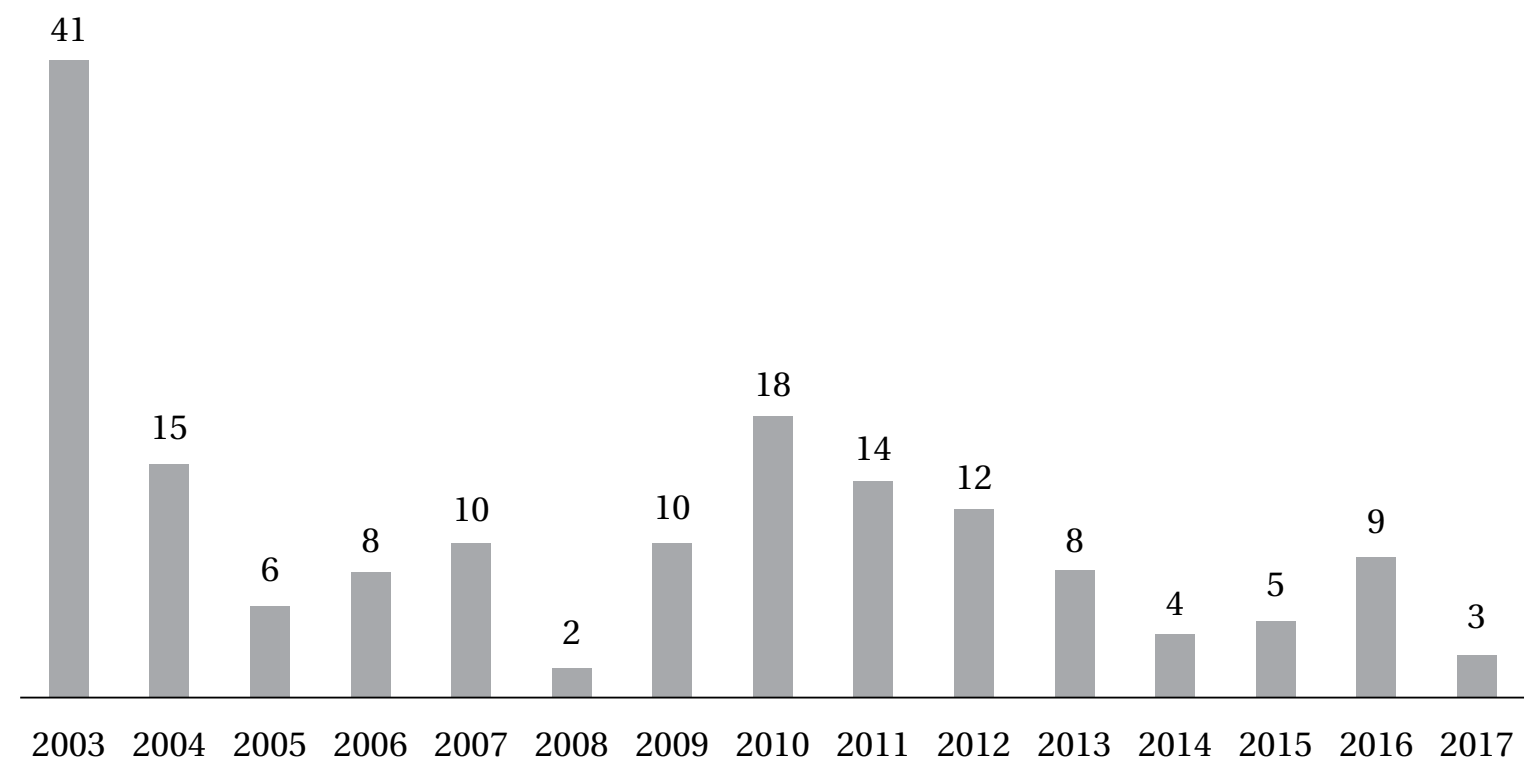

Figura

Frequência de instrumentos que tiveram parecer favorável por ano.

Para além da quantidade, nota-se uma diversidade no público-alvo para os quais os testes se destinam e também os construtos medidos pelos instrumentos disponíveis (Reppold et al. 2017a; 2017b; 2017c).

Reppold et al. (2017a) analisaram os testes psicológicos com parecer favorável no Satepsi que envolviam amostras normativas até 18 anos de idade ou que no manual estivesse explicitada a indicação para crianças e/ou adolescentes $(n=68)$. As autoras buscaram informações referentes às faixas etárias indicadas no manual, faixas etárias das amostras de padronização ou normatização, contextos indicados para uso dos testes e os construtos que os testes avaliavam. Dos instrumentos destinados exclusivamente ao público infantil ( $n=18)$, houve predominância dos testes que avaliavam o construto inteligência $(27,77 \%)$, personalidade $(16,66 \%)$ e habilidades sociais $(16,66 \%)$. Os testes voltados ao público adolescente $(\mathrm{n}=4)$ avaliavam a motivação para aprendizagem, maturidade para escolha profissional, estresse e habilidades sociais. Os testes destinados tanto ao público infantil quanto aos adolescentes $(\mathrm{n}=8)$ avaliavam autoconceito $(25,00 \%)$, personalidade $(25,00 \%)$ e inteligência $(25,00 \%)$, bem como estresse e transtorno de déficit de atenção e hiperatividade. Para os públicos adolescentes e adultos (n = 26), foram mais frequentes testes de personalidade $(34,61 \%)$ e inteligência $(26,92 \%)$. Enquanto que os testes que apresentaram estudos com amostras desde crianças até adultos $(\mathrm{n}=12)$ destinavam-se a aspectos neuropsicológicos $(41,66 \%)$ e inteligência $(25,00 \%)$.

Em outro estudo, Reppold et al. (2017b) analisaram os testes destinados para avaliação cognitiva de adultos a fim de apresentar dados sobre o construto avaliado, propriedades psicométricas do teste e a qualidade das informações contidas em seu manual. Foram selecionados os 57 testes indicados à avaliação cognitiva de adultos com parecer favorável no Satepsi em julho de 2017. Destes, 17 (29,82\%) avaliavam atenção, 16 (28,07\%) inteligência, nove (15,79\%) à avaliação do raciocínio, oito (14,03\%) mensuravam memória e sete $(12,28 \%)$ avaliavam desempenho cognitivo de forma geral. A maioria dos instrumentos evidenciava adequada relação entre os estudos psicométricos e os critérios de indicação do teste.

Com método semelhante, Reppold et al. (2017c) buscaram analisar os testes psicológicos com parecer favorável para avaliação de atributos emocionais/ sociais, de aspectos relacionados ao desenvolvimento de carreira profissional e de personalidade em adultos $(n=65)$. Do total de testes avaliados, $20(11,56 \%)$ destinavam-se à avaliação de atributos emocionais ou sociais, tais como habilidades sociais, humor/depressão, agressividade e raiva, 13 (7,51\%) construtos relacionados à orientação profissional/desenvolvimento de carreira e $32(18,50 \%)$ à avaliação do construto perso- 
nalidade, sendo 18 (10,40\%) em formato de inventário ou escala, e 14 (8,10\%) projetivos/gráficos/expressivos.

Vale a pena destacar que todos os instrumentos avaliados nos estudos descritos estavam à época com status de "favorável" no Satepsi, portanto, atenderam as especificações técnico-científicas dos requisitos mínimos obrigatórios relacionados a: (1) Fundamentação teórica; (2) objetivos do teste e contexto de aplicação, detalhando a população-alvo; (3) pertinência teórica e qualidade técnica dos estímulos utilizados nos testes; (4) evidências empíricas sobre as características técnicas dos itens do teste; (5) evidências empíricas de validade e estimativas de precisão das interpretações para os resultados do teste; (6) um sistema de correção e interpretação dos escores, podendo esse ser referenciado à norma ou não; e (7) orientações explícitas sobre aplicação e correção para que haja a garantia da uniformidade dos procedimentos. Entretanto, em diversos manuais são descritas as "qualidades psicométricas" sem necessariamente especificar o tipo de estudo realizado e, em alguns casos, o instrumento apresentou discrepância entre a faixa etária indicada e as amostras populacionais com as quais foram feitos os estudos de evidências de validade (Reppold et al., 2017a).

Sobre este aspecto, Reppold et al. (2017b) ressaltaram que carece mais cuidado quando os manuais se referem à caracterização do público-alvo, pois há discrepância e generalização entre as faixas etárias indicadas no manual e as amostras normativas. Ao falar da discrepância, as pesquisadoras se referiram às situações em que as idades limites, fossem no caso de crianças ou de idosos, tinham uma representatividade ínfima se comparada aos adultos utilizados para amostra normativa, enquanto que a generalização do público-alvo se referiam às situações em que eram adotadas expressões como "crianças", "adolescentes" ou "adultos", embora nem sempre se tenha considerado a totalidade da faixa etária correspondente a essas fases do desenvolvimento nas amostras de normatização (Reppold et al., 2017a).

A adequada discriminação do público-alvo dos testes é imprescindível para assegurar que os profissionais possam escolher qual instrumento é mais adequado para uso. Devido essa discrepância, é importante que os psicólogos fiquem atentos aos estudos normativos descritos nos manuais, bem como que os pesquisadores sejam mais prudentes no que se refere à descrição do público-alvo para qual o manual se destina.

No sentido de facilitar a identificação desses dados úteis para adequada seleção dos instrumentos, na resolução CFP no 009/2018 passou também a ser exigido que os manuais incluam uma ficha síntese informando objetivo, público-alvo, material, aplicação e correção. Além disso, observa-se que em maio de 2018, houve uma atualização do site do Satepsi que passou a fornecer informações sobre autores, editora, construto, público-alvo, idade da amostra de normatização, aplicação (coletiva/individual), correção (informatizada/ manual), data de aprovação na plenária e os prazos dos estudos de normatização e de validade para cada um dos testes que possui parecer favorável.

Considera-se um avanço a disponibilização desses dados no Satepsi na medida em que facilitará a identificação dessas informações, contribuindo para tomada de decisão sobre qual teste o profissional pode usar. Esse ajuste no site vai ao encontro das demandas de pesquisadores, que recentemente apontaram a relevância de ampliar as informações disponíveis no site para além do dado favorável ou desfavorável, possibilitando que os profissionais pudessem consultar o site para obter outras informações que são uteis na etapa de seleção dos testes psicológicos durante um processo de avaliação (Reppold et al., 2017a; 2017b; 2017c).

A partir da pré-seleção dos testes mais indicados, considerando as características do construto e do público-alvo, o psicólogo também pode consultar no Satepsi qual editora comercializa o instrumento de interesse. De acordo com as tabelas apresentadas no estudo de Reppold et al. (2017c), há um número amplo e crescente de autores e de editoras envolvidos na publicação dos instrumentos psicológicos de uso nacional. Ainda assim, observou-se que das 15 editoras que comercializavam os 180 testes psicológicos com parecer favorável em fevereiro de 2018, três delas juntas detêm o direito a comercialização de $79,32 \%$ dos testes psicológicos comercializados no Brasil.

Na comercialização dos testes psicológicos há uma representação, ainda que modesta, de laboratórios de pesquisa e empresas vinculadas a pesquisadores de Instituições de Ensino Superior que comercializam instrumentos, tais como o Instituto de Psicologia (LabPAM-UnB), em Brasília; o Laboratório de Avaliação e Medidas Psicológicas (LAMP-PUCcamp), em São Paulo; e a Empresa Distribuidora de Testes Ltda (Edites), em Minas Gerais. Ao fazer uma associação entre o ano de publicação e as editoras responsáveis pela comercialização dos testes psicológicos, nota-se que 12 dos testes comercializados pelos laboratórios de pesquisa tinham data anterior a 2003 e que a maioria dos testes publicados entre 
2004 e 2006 era oriunda de laboratórios de pesquisa. Nota-se, entretanto, que o mercado brasileiro de comercialização dos testes psicológicos passou por mudanças significativas, de modo que algumas empresas brasileiras foram adquiridas por multinacionais.

Ao analisar a lista dos 180 testes psicológicos com parecer favorável em fevereiro de 2018, verificou-se que $162(90 \%)$ são escalas, inventários, questionários e tarefas cognitivas para resolução. Para além do avanço quantitativo, nota-se que este dado reflete o progresso dos estudos na área da Psicometria, tanto da Teoria Clássica dos Testes (TCT) quanto da Teoria de Resposta ao Item (TRI). Ambas pressupõem que os testes e/ou itens são representantes legítimos, adequados e válidos do construto avaliado, e ambas têm como parâmetros importantes a validade e a precisão para legitimação das medidas.

As duas teorias também pressupõem que uma medida empírica é, necessariamente, a derivação de um construto teórico, ou seja, a medida atende ao princípio do isomorfismo, em que o estímulo (o teste ou o item) é equivalente ao traço avaliado. Diferindo-se pelo fato de que na TCT o critério avaliado é o comportamento, considerado como um representante válido do construto que está sendo mensurado (modelo comportamental). O eixo principal desta teoria está no controle da magnitude do erro da medida (para que seja o mais próximo de zero possível) e a precisão (fidedignidade) do teste como um todo (escore geral do teste) para mensurar o construto em questão. Já na TRI, o critério avaliado não é o comportamento, e sim o traço latente de cada pessoa, ou seja, sua tendência a expressar determinada conduta/característica/competência (modelo cognitivo). O principal esforço da TRI é fazer uma adequada operacionalização do construto teórico em itens válidos que representem o traço latente. Cada item, individualmente, deve ter boas propriedades psicométricas aferidas pela Curva Característica do Item (CCI), que é a capacidade de diferenciar pessoas com maior e menor habilidade/proficiência (Pasquali, 2009; Hutz, Bandeira, \& Trentini, 2015).

Os instrumentos psicométricos primam por quatro parâmetros básicos, a saber, a) padronização; b) validade; c) fidedignidade; d) normas. Com o advento do Satepsi, estas características têm sido cada vez mais aperfeiçoadas, pois observa-se que todos os instrumentos aprovados contemplam de forma explícita cada um deles:

a) A padronização define como deve ser conduzida a aplicação do teste, para quais públicos e con- textos, a forma de apurar os resultados e a forma de interpretar;

b) A validade do teste demonstra o quanto ele, de fato, é adequando para mensurar o construto (o conteúdo ou domínio) que se pretende avaliar, com a mínima margem de erro. Para isso, existem várias formas de reconhecer a validade de um instrumento, dentre elas, a validade aparente (face validity), a mais básica e mais indispensável em qualquer instrumento, a validade de construto, validade de critério (preditiva, concorrente e ecológica), validade de conteúdo, validade convergente e validade discriminante;

c) A fidedignidade do instrumento demonstra o quão preciso e confiável ele é para mensurar o construto em questão. Existem alguns métodos para reconhecer a fidedignidade do instrumento de acordo com a sua característica. Dentre elas, o método das formas alternadas ou paralelas de um teste para a mesma amostra, o método do teste-reteste, o modelo das metades (Split-half), o método de coeficientes de consistência interna (Kuder-Richardson - para escalas dicotômicas - e Alpha de Cronbach - para escalas graduadas) e o método de precisão entre avaliadores;

d) A norma técnica de um instrumento é essencial para interpretação dos resultados. A norma dá ao escore bruto de um indivíduo avaliado um sentido, um significado, uma interpretação. Fornece uma informação acerca da posição deste indivíduo em relação à característica que está sendo avaliada. São dois os principais sistemas de normas técnicas: intragrupo e de conteúdo ou critério. No primeiro caso, as normas técnicas são tão boas quanto mais amplos são os grupos amostrais e quanto maior é o número de pessoas avaliadas em cada subamostra (sexo, idade, escolaridade, nível socioeconômico, região do país). Nesse sistema, se identifica quanto o desempenho de uma pessoa se afasta da média do seu grupo de referência, seja para mais ou para menos. No segundo caso, sistema normativo com referência ao conteúdo ou critério, a interpretação do desempenho de uma pessoa é feita com base num ponto de corte proposto para o instrumento, de acordo com o embasamento teórico do sistema. Nesse caso, as normas técnicas são tão boas quanto melhores são os parâmetros de acurácia, especificidade e sensibilidade do instrumento, que permitem minimizar os casos de resultados falsos positivos efalsos negativos. 
A Resolução nº 009/2018 estabelece os critérios para classificar estes parâmetros psicométricos como "excelente", "bom", "suficiente" e "insuficiente".

Voltando à lista dos 180 testes psicológicos com parecer favorável no Satepsi em fevereiro de 2018, 18 (10\%) eram métodos projetivos (Tabela 1). De acordo com Reppold et al. (2017c), a quantidade de projetivos com parecer favorável para uso merece destaque, sugerindo um avanço para área e evidenciando que a complexidade dos métodos projetivos não prescinde do rigor psicométrico.

Reppold et al. (2017c) também destacaram que a maioria dos testes projetivos disponíveis são os de manchas de tinta, como os métodos de Rorschach e Zulliger, seguidos pelos Testes de Apercepção Temática e suas variações. Nesse sentido, cabe explicitar uma particularidade dos métodos projetivos em relação aos demais instrumentos. Durante a análise dos métodos projetivos, considera-se o sistema interpretativo apresentado como instrumentos independentes. A título de exemplo, o método de Rorschach possui no Brasil cinco sistemas interpretativos com parecer favorável, sendo eles: o Rorschach - Sistema Compreensivo, o Rorschach Clínico Sistema Aníbal Silveira, O Rorschach: Teoria e Desempenho - Sistema Klopfer, o Rorschach - Sistema da Escola Francesa, Rorschach - Sistema de Avaliação por Desempenho (R-PAS). Deste modo, cada sistema interpretativo deve ter suas próprias evidências de validade e consistência da interpretação para ser considerado favorável.

Destaca-se que embora o Rorschach tenha cinco sistemas interpretativos com parecer favorável, todos utilizam o mesmo estímulo. Sobre isso, cabe ressaltar que o profissional que usa os métodos projetivos deve ficar atento à qualidade gráfica do material usado na administração, visando preservar as características dos estímulos. No caso do Rorschach, por exemplo, recentemente houve denúncia ao CFP que estariam sendo comercializados estímulos diferentes dos originais (CFP, 2017). O uso de material diferente do orginalmente estudado é considerado um problema ético, pois as características da coloração e sombreamento das manchas de tinta interferem diretamente na percepção do examinando. Essa situação é bastante grave na medida em que todos os parâmetros interpretativos e normativos foram estabelecidos considerando as características do estímulo. Nota-se, deste modo, que preservar a qualidade gráfica dos cartões, seja do Rorschach ou de qualquer outro método projetivo, é imprescindível.

Na Tabela 1, verifica-se que três métodos projetivos foram aprovados em 2003. No que se refere ao primeiro da lista, a Técnica de Zulliger (Z-teste), novos estudos tiveram parecer favorável em 2016, ampliando o uso para administração tanto coletiva quanto individual. Para que os demais se mantenham na lista de

Tabela 1

Métodos Projetivos em função do ano de publicação com data de aprovação.

\begin{tabular}{lcc}
\hline Instrumento & Ano & Aprovação \\
\hline Técnica de Zulliger (Z-Teste) - Sistema Klopfer - Forma coletiva & 1999 & $10 / 12 / 03$ \\
Rorschach - Sistema Compreensivo & 1999 & $25 / 10 / 03$ \\
Teste de Apercepção Temática (TAT) & 2003 & $25 / 10 / 03$ \\
Casa - Árvore - Pessoa (HTP) & 2003 & $16 / 01 / 04$ \\
Rorschach Clínico - Sistema Aníbal Silveira & 2007 & $13 / 03 / 04$ \\
Teste Contos de Fadas (FTT) & $\mathrm{S} / \mathrm{inf}$. & $04 / 06 / 04$ \\
Rorschach - Sistema da Escola Francesa & 2000 & $22 / 01 / 05$ \\
As Pirâmides Coloridas de Pfister (TPC) & 2005 & $22 / 01 / 05$ \\
Teste de Fotos de Profissão (BBT) & 2000 & $13 / 08 / 05$ \\
Teste de Fotos de Profissão (BBT) - BBT-Br Feminino & 2004 & $13 / 08 / 05$ \\
O Rorschach: Teoria e Desempenho - Sistema Klopfer & 2004 & $12 / 05 / 06$ \\
Teste de Zulliger - Sistema Compreensivo (ZSC) - Individual & 2009 & $31 / 10 / 08$ \\
Teste de Apercepção Infantil (CAT-A) - Figuras de Animais & 2013 & $22 / 03 / 13$ \\
Técnica de Apercepção para Idosos (SAT) & 2012 & $24 / 05 / 13$ \\
As Pirâmides Coloridas de Pfister (TPC) - Crianças e Adolescentes & 2014 \\
Técnica de Zulliger (Z-Teste) - Sistema Klopfer - Forma coletiva e Individual & 2016 & $30 / 11 / 13$ \\
Teste de Apercepção Infantil (CAT-H) - Figuras Humanas & 2016 \\
Rorschach - Sistema de Avaliação por Desempenho (R-PAS) & 2016 & $19 / 08 / 16$ \\
\hline
\end{tabular}


instrumentos com parecer favorável será preciso que seus responsáveis técnicos submetam novos estudos a serem analisados. Caso haja mais interesse em informações sobre cada um dos métodos projetivos listados na Tabela, sugere-se a leitura de Villemor-Amaral e Werlang (2008) e de Hutz, Bandeira e Trentini (2018).

Nos manuais mais recentes, seja de métodos projetivos ou psicométricos, há um detalhamento mais amplo das informações, bem como uma quantidade maior de estudos psicométricos (Reppold et al., 2017a). Assim, é visível a melhoria na qualidade, de um modo geral, dos manuais dos testes na última década. Não se pode, com isso, conforme apontado por Noronha e Reppold (2010, p.196), "ingenuamente concluir que a avaliação psicológica brasileira se encontra em nível de excelência e pode ser equiparada à das grandes potências internacionais, com sua centena de testes". $\mathrm{O}$ caminho trilhado até o momento sugere que o Satepsi tem possibilitado avanços para área, sendo necessário dar prosseguimento à melhoria dos testes psicológicos e concomitante a essas ações deve-se pensar continuamente em estratégias para qualificação dos psicólogos.

\section{Referências}

Associação Nacional de Pesquisa e Pós-graduação em Psicologia - ANPEPP. (2018). Gt's em situação regular. Recuperado de http://www.encurtador.com.br/rsyFQ

Conselho Federal de Psicologia - CFP. (2007). Teste psicológico: vitória para o CFP. Brasília, DF: o autor.

Conselho Federal de Psicologia - CFP. (2017). Parecer No 1/2017/Gtec/Cg. Comercialização das pranchas de Rorschach sem comprovação científica e sem aprovação do CFP. Brasília, DF: o autor.

Fórum de Entidades Nacionais da Psicologia Brasileira - FENPB. (2018). Sobre o FENPB. Recuperado de http:// www.fenpb.org/sobre/

Hutz, C. S., \& Bandeira, D. R. (2003) Avaliação psicológica no Brasil: Situação atual e desafios para o futuro. In: O. H. Yamamoto, \& V. V. Gouveia (Eds.), Construindo a psicologia brasileira: desafios da ciência e prática psicológica (pp.261-275). São Paulo, SP: Casa do Psicólogo.

Hutz, C. S., Bandeira, D. R., \& Trentini, C. M. (2015). Psicometria. Porto Alegre, RS: Artmed.

Hutz, C. S., Bandeira, D. R., \& Trentini, C. M. (2018). Avaliação psicológica da inteligência e da personalidade. Porto Alegre, RS: Artmed.

Instituto Brasileiro de Avaliação Psicológica - IBAP. (2015). Catálogo dos laboratórios de avaliação psicológica no Brasil. São Paulo, SP: o autor. Recuperado em http://www.ibapnet.org.br/congresso2015/arquivos

Noronha, A. P. P. (2002). Análise de testes de personalidade: qualidade do material, das instruções, da documentação e dos itens qualidade de testes de personalidade. Estudos de Psicologia (Campinas), 19(3), 55-65. doi: 10.1590/ S0103-166X2002000300006

Noronha, A. P. P, Primi, R., \& Alchieri, J. C. (2004) Parâmetros psicométricos: uma Análise de testes psicológicos comercializados no Brasil. Psicologia Ciência e Profissão, 24(4), 88-99. https://doi.org/10.1590/S1414-98932004000400011.

Noronha, A. P. P., \& Reppold, C. T. (2010). Considerações sobre a avaliação Psicológica no Brasil. Psicologia: Ciência e Profissão, 30(spe), 192-201. https://doi.org/10.1590/S1414-98932010000500009

Noronha, A. P. P., Sartori, F. A., Freitas, F. A., \& Ottati, F. (2001). Informações contidas nos manuais de testes de inteligência publicados no Brasil. Psicologia em Estudo, 6(2), 101-106. https://doi.org/10.1590/S1413-73722001000200014

Nunes, M. F. O., Muniz, M., Reppold, C. T., Faiad, C., Bueno, J. M. H., \& Noronha, A. P. P. (2012). Diretrizes para o ensino de avaliação psicológica. Avaliação Psicológica, 11(2), 309-316.

Pasquali, L. (2009). Psicometria. Revista Escola de Enfermagem da USP, 43(Esp), 992-999. https://doi.org/10.1590/ S0080-62342009000500002.

Primi, R. (2010). Avaliação psicológica no Brasil: Fundamentos, situação atual e direções para o futuro. Psicologia: Teoria e Pesquisa, 26(spe), 25-35.: https:// dx.doi.org/10.1590/S0102-37722010000500003

Primi, R., \& Nunes, C. H. S. (2010). O SATEPSI: Desafios e propostas de aprimoramento. In Conselho Federal de Psicologia. Avaliação psicológica: Diretrizes na regulamentação da profissão (pp. 129-148). Brasília, DF: o autor.

Reppold, C. T., Serafini, A. J., Gurgel, L. G., \& Kaiser, V. (2017b). Avaliação de aspectos cognitivos em adultos: análise de manuais de instrumentos aprovados. Avaliação Psicológica, 16(2), 137-144. https://dx.doi.org/10.15689/AP.2017.1602.03 
Reppold, C. T., Serafini, A. J., Magnan, E. S., Damion, M., Kaiser, V., \& Almeida, L. (2017c). Análise de manuais de testes psicológicos aprovados pelo SATEPSI para adultos. Psicologia Teoria e Prática, 16(1), 19-28. http://dx.doi. org/10.15689/ap.2017.1601.03

Reppold, C. T., Serafini, A. J., Ramires, D. A., \& Gurgel, L. G. (2017a). Análise dos manuais psicológicos aprovados pelo SATEPSI para avaliação de crianças e adolescentes no Brasil. Avaliação Psicológica, 16(1), 19-28. https:// dx.doi.org/10.15689/ap.2017.1601.03.

Resolução No 002, de 23 de março de 2003. Define e regulamenta o uso, a elaboração e a comercialização de testes psicológicos e revoga a Resolução CFP n 025/2001. Brasília, DF: Conselho Federal de Psicologia.

Resolução No 009, de 25 de abril de 2018. Estabelece diretrizes para a realização de Avaliação Psicológica no exercício profissional da psicóloga e do psicólogo, regulamenta o Sistema de Avaliação de Testes Psicológicos - SATEPSI e revoga as Resoluções n 002/2003, no 006/2004 e n 005/2012 e Notas Técnicas nº 01/2017 e 02/2017. Brasília, DF: Conselho Federal de Psicologia.

Santos, A. M., Anache, A. A., \& Santana, R. C. (2015). Overview of Brazilian Scientific Production in Psychological Evaluation. Psico-USF, 20(3), 547-559. https://dx.doi.org/10.1590/1413-82712015200315

Villemor-Amaral, A. E., \& Werlang, B. S. G. (2008) Atualizações em métodos projetivos para avaliação psicológica. São Paulo, SP: Casa do Psicólogo.

\section{Lucila Moraes Cardoso}

Profa. Adjunta do curso de Psicologia e Programa de Pós-graduação em Educação da Universidade Estadual do Ceará (UECE) e Profa. Colaboradora no Programa de Pós-graduação em Psicologia da Universidade Federal do Ceará (UFC). E-mail: lucila.cardoso@uece.br

\section{José Humberto da Silva-Filho}

Prof. Adjunto do curso de Psicologia e Programa de Pós-graduação em Psicologia, na Universidade Federal do Amazonas (UFAM).

E-mail: zehumberto@uol.com.br

Endereço para envio de correspondência:

A/C Lucila Moraes Cardoso - Curso de Psicologia da Universidade Estadual do Ceará - UECE - Av. Dr. Silas Munguba, 1700, Campus do Itaperi, Fortaleza-CE - CEP: 60.714 .903

Recebido: $23 / 07 / 2018$

Aprovado: 08/08/2018

Received: 07/23/2018

Approved: 08/08/2018

Recibido: 23/07/2018

Aceptado: 08/08/2018

Como citar: Cardoso, L. M., \& Silva-Filho, J. H. (2018). Satepsi e a Qualidade Técnica dos Testes Psicológicos no Brasil. Psicologia: Ciência e Profissão, 38(n.spe), 40-49. https://doi.org/10.1590/1982-3703000209112

How to cite: Cardoso, L. M., \& Silva-Filho, J. H. (2018). Satepsi and the Technical Quality of Psychological Testing in Brazil. Psicologia: Ciência e Profissão, 38(n.spe), 40-49. https://doi.org/10.1590/1982-3703000209112

Cómo citar: Cardoso, L. M., \& Silva-Filho, J. H. (2018). Satepsi y la calidad técnica de las pruebas psicológicas en Brasil. Psicologia: Ciência e Profissão, 38(n.spe), 40-49. https://doi.org/10.1590/1982-3703000209112 